\title{
Eventos Estressores no Contexto Acadêmico: Uma Breve Revisão da Literatura Brasileira
}

\author{
Marucia Patta Bardagi ${ }^{\text {a,* }}$, \& Claudio Simon Hutz ${ }^{\text {b }}$ \\ ${ }^{a}$ Universidade Federal de Santa Catarina, Florianópolis, Brasil, \\ \& buniversidade Federal do Rio Grande do Sul, Porto Alegre, Brasil
}

\begin{abstract}
RESUMO
Os estudos sobre percepção de estressores e estratégias de enfrentamento no contexto do trabalho apontam o impacto negativo destes sobre o desempenho e o bem-estar psicológico. Entretanto, há poucas pesquisas sobre o tema no período universitário. Este estudo realizou uma revisão da literatura sobre estresse e coping no contexto acadêmico para identificar as contribuições dos estudos na compreensão das trajetórias acadêmicas e para a criação de intervenções preventivas aos problemas percebidos pelos estudantes. Nos estudos encontrados, houve predomínio de pesquisas na área da saúde, diversidade de eventos percebidos como estressores pelos alunos, e uso de estratégias mais focadas na emoção. Os resultados indicam a importância da ampliação de pesquisas na área e da avaliação dos contextos específicos de formação.
\end{abstract}

Palavras-chave: eventos estressores; estudantes universitários; coping.

\section{ABSTRACT \\ Stressors in the Academic Context: A Brief Review of Brazilian Literature}

Studies about the perception of stressors and coping strategies in the work context point out the stressors' negative impact on performance and psychological well-being. However, there is little research on this topic during the college period. This study consists in a literature review about stress and coping in the academic context, in order to identify the contribution of these studies for the understanding of academic paths; and develop preventive interventions directed to the students' perceived problems. Results found a majority of studies in health fields, a variety of events perceived as stressful by students, and the use of strategies specially focused on emotions. These results indicate the importance of further research in the area and of the assessment of specific contexts of study.

Keywords: stressor events; university students; coping.

A forma como são percebidas as situações adversas relativas à carreira e os modos empregados pelos indivíduos para lidar com elas configuram, há tempos, um campo fundamental de investigação na área do desenvolvimento vocacional no âmbito internacional (Heppner, Cook, Wright, \& Johnson, 1995; Jackson \& Finney, 2002; Lent, Brown, Talleyrand, McPartland, Davis e cols., 2002; Misra \& McKean, 2000; Robitschek \& Cook, 1999). No Brasil, entretanto, são mais frequentes estudos sobre percepção de estressores e estratégias de coping em contextos ocupacionais específicos, avaliando sintomatologia de estresse, aspectos do trabalho que favorecem o estresse e a forma como os profissionais de diferentes áreas lidam com o estresse no trabalho (Dela Coleta \& Dela Coleta, 2008; Lipp \& Tanganelli, 2002; Pinheiro, Tróccoli, \& Tamayo, 2003; Rodrigues \& Chaves, 2008; Rossetti, Ehlers, Guntert, Sá Leme e cols., 2008; Suehiro, Santos, Hatamoto, \& Cardoso, 2008; Telles \& Pimenta, 2009, entre outros). Já a literatura nacional sobre avaliação

Endereço para correspondência: Marucia Patta Bardagi, Rua João Pio Duarte Silva, 180, 103 B, Bairro Córrego Grande, Florianópolis, SC, 88037-000. E-mail: marucia.bardagi@gmail.com. 
de estressores e coping entre estudantes universitários e/ou focando as questões de carreira é menos frequente.

Inicialmente, é necessário definir e descrever o que são eventos estressores e estratégias de coping. $\mathrm{O}$ modelo transacional do estresse, também denominado por alguns autores de modelo interacionista cognitivo (Folkman, Lazarus, Gruen, \& De Longis, 1986; Lazarus \& Folkman, 1984) é um dos mais utilizados para abordar as relações entre o indivíduo e as situações adversas que ele enfrenta. Os trabalhos de referência a partir dessa perspectiva, utilizada neste estudo, definem os eventos estressores como todo e qualquer acontecimento, eventual ou sistemático, que desafia os limites e recursos psicológicos do sujeito para lidar com ele (Lazarus \& Folkman, 1984). Para tanto, o indivíduo avalia cognitivamente aspectos como a controlabilidade, a previsibilidade e a extensão do evento. Quanto mais incontrolável ou imprevisível for um evento, maior sua probabilidade de ser percebido como estressante, uma vez que antecipar a ocorrência de um evento estressante permite que se inicie um processo preparatório que reduz os efeitos nocivos do acontecimento. No entanto, mesmo situações que são controláveis e/ou previsíveis podem ser percebidas como estressantes quando exigem muito do indivíduo ou desafiam sua autopercepção de capacidade.

Já as estratégias de coping são os esforços cognitivos e comportamentais que os indivíduos despendem na tentativa de lidar com os estressores, ou seja, controlar ou reduzir os danos causados por eles (Folkman e cols., 1986; Lazarus \& Folkman, 1984). O coping é uma resposta com o objetivo de aumentar, criar ou manter a percepção de controle pessoal sobre as situações vivenciadas. Ao perceber uma situação como estressante, os indivíduos costumam experimentar uma sensação emocional desagradável, e tendem a adotar estratégias que permitam minimizar seu sofrimento (Folkman \& Lazarus, 1980).

A classificação dos tipos de estratégias de coping não apresenta um consenso (Pinheiro e cols., 2003). No entanto, comumente, dento da perspectiva interacionista/transacional, as estratégias são amplamente divididas entre estratégias focadas no problema e estratégias focadas na emoção (Chaves, Cadê, Montovani, Leite, \& Spire, 2000; Folkman \& Lazarus, 1980; Seidl, Tróccoli, \& Zannon, 2001). As primeiras referem-se a estratégias mais voltadas à realidade e, portanto, mais adaptativas, pois são capazes de modificar as pressões ambientais. A pessoa engaja-se no manejo ou modificação do problema tentando controlar ou lidar com a ameaça, tais como a solução de problemas e o planejamento. O coping centrado na emoção teria como função principal a regulação da resposta emocional causada pelo problema/estressor com o qual a pessoa se defronta, podendo representar atitudes de afastamento ou paliativas em relação à fonte de estresse, como negação ou esquiva. Este tipo de coping não modifica a situação propriamente dita, mas serve para o indivíduo negociar com as emoções, e assim, manter uma autoestima positiva. Essas estratégias não são necessariamente excludentes: diferentes estratégias de enfrentamento podem ser utilizadas simultaneamente para lidar com determinada situação estressora.

Embora as diferenças de gênero não sejam unânimes na literatura, e alguns estudos não encontrem diferenças significativas entre homens e mulheres para a maioria das estratégias (Seidl e cols., 2001), algumas pesquisas tendem a apontar um número maior de estratégias utilizadas entre as mulheres em comparação com os homens; em particular, elas tenderiam a utilizar mais estratégias centradas na emoção e de busca de apoio (Hansen \& Jarvis, 2000; Olah, 1995; Piko, 2001). Ao se analisar a natureza dos eventos, vêse que, por exemplo, em crianças, estratégias de ação agressiva e de busca de apoio social ocorrem com maior frequência em conflitos com pares, enquanto que com adultos ou outras figuras de autoridade são mais comuns estratégias de evitação, aceitação ou inação, além de expressão emocional quando a negociação não é possível (Dell'Aglio \& Hutz, 2002). Jo Lohman e Jarvis (2000) acharam diferenças nas estratégias de coping utilizadas no contexto familiar em comparação a outros contextos, mostrando que no primeiro são mais comuns estratégias centradas na emoção. Os estudos sobre percepção de estressores e coping nos contextos de trabalho no Brasil, não apontam consenso quanto às diferenças de gênero na presença de estresse. Quanto às estratégias de enfrentamento, os estudos nacionais também são incongruentes; alguns mostram predomínio de estratégias focadas na emoção (Dela Coleta \& Dela Coleta, 2008; Rodrigues \& Chaves, 2008) enquanto outros apontam maior uso de estratégias focadas no problema (Seidl e cols., 2001; Telles \& Pimenta, 2009), indicando que talvez o contexto específico de trabalho seja determinante para a escolha do coping.

Pode-se pensar o período da formação universitária também como um momento de vulnerabilidade ao 
estresse. O mesmo caracteriza-se como um período desafiador ao aluno e que impõe tarefas complexas a enfrentar nos domínios acadêmico (novos ritmos e estratégias de aprendizagem e novos sistemas de ensino e avaliação), social (novos padrões de relacionamento, além de ampliação da rede social), pessoal (estabelecimento de um sentido mais forte de identidade) e vocacional (definição de metas de carreira) (Almeida \& Soares, 2003). Ross, Niebling e Heckert (1999), utilizando a escala The Student Stress Survey para determinar as maiores fontes de estresse entre universitários, observaram que $38 \%$ das fontes de estresse citadas eram relativas ao âmbito intrapessoal, $28 \%$ ao ambiental, $19 \%$ ao interpessoal e $15 \%$ ao nível acadêmico. Misra e McKean (2000) observaram que as mulheres tendem a apresentar maior nível de estresse acadêmico do que os homens e que a ansiedade e a dificuldade de organização do tempo são os principais preditores do estresse acadêmico. Ainda, os resultados de seu estudo com estudantes universitários americanos indicam que os estudantes mais novos, de primeiro e segundo ano, foram os que apresentaram maior nível de estresse.

Os estudos internacionais na área do desenvolvimento vocacional costumam avaliar, ainda, as estratégias de coping em relação à tomada de decisão de carreira. Heppner e cols. (1995) identificaram três tipos de estratégias utilizadas por alunos de todos os níveis ao lidar com questões vocacionais: a) coping reflexivo, tendência a emitir comportamentos para analisar as relações causais entre os eventos, planejar ações e ser sistemático ao lidar com as situações; b) coping reativo, tendência a apresentar respostas emocionais ou cognitivas que reduzem ou distorcem os comportamentos de coping; e c) coping supressivo, tendência a negar a existência de problemas e evitar os comportamentos de coping. Em seu estudo com universitários, Robitschek e Cook (1999) observaram que há necessidade de desenvolver maiores níveis de coping reflexivo nos alunos, uma vez que há maior tendência a utilizar estratégias reativas. Nesse sentido, outro estudo já apontou as relações entre coping evitativo (ou supressivo) e menor ajustamento emocional de estudantes, com índices elevados de ansiedade e depressão; e coping ativo (ou reflexivo) e maior ajustamento (Crockett, Iturbide, Torres-Stone, McGinley, Raffaelli, \& Carlo, 2007).

Eagan e Walsh (1995) compararam estudantes com escolhas congruentes, incongruentes e indecisas de carreira e encontraram diferenças de gênero, em que as mulheres, de forma geral, utilizam mais estratégias de busca de apoio e evitação do que os homens; quanto ao tipo de escolha, alunos com escolhas mais congruentes tendem a utilizar menos estratégias de evitação. Jackson e Finney (2002) apontam diferença de idade nas estratégias de coping de estudantes universitários. Ao lidar com eventos estressores (especialmente conflitos com os pares), os alunos mais novos tendem a apresentar maior sofrimento psicológico e a utilizar estratégias mais ineficazes e centradas na emoção do que alunos mais velhos. Em um estudo qualitativo onde foram entrevistados estudantes de duas universidades americanas, Lent e cols. (2002) avaliaram as estratégias utilizadas para lidar com as barreiras percebidas de carreira; os resultados apontam a utilização de estratégias centradas no problema, busca de apoio e reestruturação cognitiva como as mais frequientes, enquanto a busca de ajuda profissional não foi citada com frequência; ainda, as principais barreiras de carreira (e fontes de estresse) percebidas pelos alunos pesquisados foram contextuais, como dificuldades financeiras e influências negativas de amigos ou família.

No entanto, para investigar as estratégias de enfrentamento e suas consequências no âmbito universitário, é preciso um mapeamento dos eventos estressores, algo pouco frequente nos estudos nacionais. Avaliar os estressores vocacionais/acadêmicos mostra-se importante, uma vez que estudos têm apontado a população universitária como vulnerável ao estresse crônico e ao burnout (Martínez \& Pinto, 2005; Martínez, Pinto, \& Silva, 2000), assim como as condições de lidar com as barreiras e dificuldades durante a formação têm sido apontadas como favorecedoras do desenvolvimento de carreira entre universitários. Martinez e Pinto (2005), avaliando alunos de Portugal e Espanha, encontraram associação positiva entre burnout e tendência à evasão e associações negativas entre burnout e desempenho acadêmico e expectativa de sucesso. Tanto para as unidades de ensino quanto para os serviços de atendimento aos alunos universitários, identificar as principais fontes de preocupação e ansiedade relativas ao contexto de formação superior pode auxiliar no combate à evasão e ao desengajamento dos alunos, aumentando as possibilidades de satisfação com o curso e a profissão. Nesse sentido, o objetivo deste trabalho é contribuir para a sistematização das informações sobre o tema, a partir da apresen- 
tação de uma revisão da literatura brasileira sobre estresse e estratégias de coping em estudantes universitários, a partir do mapeamento de publicações sobre o tema no período de 1999 a 2009. Com isso, pretende-se identificar os avanços e necessidades da área e auxiliar a elaboração de novos estudos sobre o tema no contexto universitário.

\section{MÉTODO}

Para esta revisão, procedeu-se a uma busca em bases de dados eletrônicas (SciELO e BVS-Psi), utilizando combinações duas a duas entre, de um lado as palavras-chave estresse, eventos estressores, estressores acadêmicos, coping e estratégias de enfrentamento, e de outro as palavras-chave ensino superior, estudante(s) universitário(s), universidade, carreira e adulto(s) jovem(s). O ano de 1999 foi escolhido como inicial em função de ter havido, a partir dele, um crescimento das publicações sobre estresse em geral no Brasil (Noronha \& Fernandes, 2008) e as bases de dados foram escolhidas por serem consideradas as mais completas para o acesso à produção nacional. Foram considerados para análise os artigos cujos temas fossem relativos à discussão (no caso de estudos teóricos) ou avaliação de presença de estresse ou situações consideradas geradoras de estresse ou ansiedade entre alunos do ensino superior. Foram excluídos os artigos que tivessem como foco outros públicos uni- versitários como professores ou funcionários, aqueles que avaliavam especificamente a Síndrome de Burnout e não estresse, aqueles que avaliavam apenas estilos de coping em geral, sem relacioná-los a questões do contexto acadêmico e também aqueles que avaliassem eventos estressores não pertinentes ao contexto acadêmico, como doenças, problemas familiares, etc. Em função do número de artigos obtidos não ser excessivo, procedeu-se uma análise descritiva dos mesmos, apresentando seus principais resultados e características (amostra, tipo de análise, uso ou não de instrumentos, etc).

\section{RESULTADOS}

Foram encontrados 12 artigos sobre o tema, sendo um artigo de revisão, sete artigos sobre avaliação de sintomatologia de estresse em alunos do Ensino Superior e seis que avaliaram (juntamente ou não à sintomatologia) eventos estressores e/ou coping no contexto universitário. As Tabelas 1 e 2 apresentam as principais características de cada trabalho. Observa-se que grande parte dos estudos (8) foca alunos da área de saúde, como medicina e enfermagem e que a avaliação de estresse se dá principalmente pelo uso do $I S S L$ - Inventário de Sintomas de Stress para Adultos. Ainda, há predomínio de estudos quantitativos e um aumento de produção a partir do ano de 2007.

Tabela 1

Descrição dos Artigos Revisados Sobre Estresse no Contexto Universitário no Período 1999-2006

\begin{tabular}{|c|c|c|c|c|}
\hline & Ano & Tipo de estudo & Amostra & $\begin{array}{l}\text { Instrumentos para avaliação de } \\
\text { estresse ou eventos estressores }\end{array}$ \\
\hline Baptista \& Campos & 2000 & $\begin{array}{l}\text { Empírico } \\
\text { quantitativo }\end{array}$ & 52 alunos de Psicologia & ISSL \\
\hline Calais, Andrade \& Lipp & 2003 & $\begin{array}{l}\text { Empírico } \\
\text { quantitativo }\end{array}$ & $\begin{array}{l}295 \text { estudantes de Ensino } \\
\text { Médio, pré-vestibular e } \\
\text { Ensino Superior de várias } \\
\text { áreas }\end{array}$ & ISSL \\
\hline Furtado, Falcone \& Clark & 2003 & $\begin{array}{l}\text { Empírico } \\
\text { quantitativo }\end{array}$ & 178 alunos de Medicina & ISSL \\
\hline $\begin{array}{l}\text { Evangelista, Hortense \& } \\
\text { Souza }\end{array}$ & 2004 & $\begin{array}{l}\text { Empírico } \\
\text { experimental }\end{array}$ & 15 alunos de Enfermagem & $\begin{array}{l}\text { Escala de tarefas acadêmicas e } \\
\text { métodos psicofísicos de estimação de } \\
\text { magnitudes e emparelhamento inter- } \\
\text { modal }\end{array}$ \\
\hline Zonta, Robles \& Grosseman & 2006 & $\begin{array}{l}\text { Empírico } \\
\text { qualitativo }\end{array}$ & 25 alunos de Medicina & Entrevista \\
\hline
\end{tabular}


Tabela 2

Descrição dos Artigos Revisados Sobre Estresse no Contexto Universitário no Período 2007-2009

\begin{tabular}{|c|c|c|c|c|}
\hline & Ano & Tipo de estudo & Amostra & $\begin{array}{l}\text { Instrumentos para avaliação de } \\
\text { estresse ou eventos estressores }\end{array}$ \\
\hline $\begin{array}{l}\text { Calais, Carrara, Brum, } \\
\text { Batista, Yamada \& Oliveira } \\
\end{array}$ & 2007 & $\begin{array}{l}\text { Empírico } \\
\text { quantitativo }\end{array}$ & $\begin{array}{l}105 \text { estudantes de } \\
\text { Jornalismo }\end{array}$ & ISSL \\
\hline Santos \& Alves Junior & 2007 & $\begin{array}{l}\text { Empírico } \\
\text { quantitativo }\end{array}$ & $\begin{array}{l}27 \text { mestrandos de } \\
\text { Ciências da Saúde }\end{array}$ & $\begin{array}{l}\text { ISSL, Escala de modos de enfren- } \\
\text { tamento de problemas e um } \\
\text { questionário sobre estressores }\end{array}$ \\
\hline Bondan \& Bardagi & 2008 & $\begin{array}{l}\text { Empírico } \\
\text { quantitativo }\end{array}$ & $\begin{array}{l}86 \text { estudantes universitá- } \\
\text { rios de várias áreas }\end{array}$ & $\begin{array}{l}\text { Escala de eventos estressores } \\
\text { acadêmicos }\end{array}$ \\
\hline $\begin{array}{l}\text { Aguiar, Vieira, Vieira, Aguiar } \\
\& \text { Nóbrega }\end{array}$ & 2009 & $\begin{array}{l}\text { Empírico } \\
\text { quantitativo }\end{array}$ & $\begin{array}{l}200 \text { estudantes de } \\
\text { Medicina }\end{array}$ & ISSL \\
\hline Costa \& Polak & 2009 & $\begin{array}{l}\text { Construção e } \\
\text { validação de } \\
\text { instrumento }\end{array}$ & $\begin{array}{l}160 \text { estudantes de } \\
\text { Enfermagem }\end{array}$ & $\begin{array}{l}\text { AEEE - Avaliação de estresse em } \\
\text { estudantes de enfermagem }\end{array}$ \\
\hline $\begin{array}{l}\text { Gonçalves \& Benevides- } \\
\text { Pereira }\end{array}$ & 2009 & $\begin{array}{l}\text { Revisão da } \\
\text { literatura }\end{array}$ & $\begin{array}{l}\text { Estudos com estudantes } \\
\text { de Medicina }\end{array}$ & Não se aplica \\
\hline $\begin{array}{l}\text { Katsurayama, Becker, } \\
\text { Gomes, Santos, Makimoto \& } \\
\text { Santana }\end{array}$ & 2009 & $\begin{array}{l}\text { Empírico } \\
\text { quantitativo }\end{array}$ & 61 alunos de Medicina & $\begin{array}{l}\text { ISSL e questionário para avaliação } \\
\text { de aspectos pessoais, familiares e } \\
\text { acadêmicos }\end{array}$ \\
\hline
\end{tabular}

No artigo de revisão de Gonçalves e BenevidesPereira (2009), que descreve uma revisão de estudos sobre saúde mental de universitários e sobre educação médica no Brasil, aspectos como lócus de controle interno, resiliência e compromisso com a carreira podem funcionar como moderadores do estresse entre estudantes. Já entre os fatores causadores de estresse, ao menos em estudos com estudantes de medicina, estão citados como principais a falta de tempo livre e a dificuldade de relacionamento com professores. As autoras enfatizam a importância de pesquisas sobre saúde psicológica de estudantes em geral, além do mapeamento de eventos estressores e a criação de serviços de apoio aos alunos. Katsurayama e cols. (2009) apontam também o risco que o alto nível de exigência e a dificuldade de expressar sentimentos de alguns alunos podem trazer para o desenvolvimento do estresse. A criação de políticas institucionais que promovam condições favoráveis a um melhor desenvolvimento das potencialidades acadêmicas dos alunos foi citada pelas autoras e também por Calais e cols. (2007).

Quanto à avaliação geral de estresse em estudantes, os estudos costumam apontar prevalência de sintomas entre as mulheres (Aguiar, Vieira, Vieira, Aguiar, \& Nóbrega, 2009; Calais, Andrade, \& Lipp, 2003; Calais e cols., 2007; Furtado, Falcone, \& Clark, 2003; Santos $\&$ Alves Junior, 2007); quanto ao período no curso, alguns resultados apontam maior estresse em alunos de início de curso (Furtado e cols., 2003) enquanto outros apontam predomínio do estresse no final de curso (Calais e cols., 2003, 2007). Na comparação com alunos de outros níveis escolares, Calais e cols. (2003) observaram que os alunos universitários tinham menor nível de estresse do que alunos de prévestibular e alunos do $3^{\circ}$ ano do Ensino Médio. Nos estudos que avaliaram o percentual de alunos com alguma sintomatologia de estresse, a partir do uso do ISSL (Inventário de Sintomas de Estresse para Adultos), esses índices variaram entre $40 \%$ e $65 \%$ das amostras pesquisadas (Aguiar e cols., 2009; Calais e cols., 2007; Katsurayama e cols., 2009; Santos \& Alves Junior, 2007).

Quanto à sintomatologia, os estudos apontam predomínio de sintomas psicológicos do estresse em detrimento dos sintomas físicos (Aguiar e cols., 2009; Calais e cols., 2003); ao fazer uma diferenciação em relação ao momento do curso, Calais e cols. (2007) encontraram mais frequiência de sintomas físicos entre calouros e mais frequiência de sintomas psicológicos entre alunos de final de curso. Já Baptista e Campos (2000), ao avaliar alunos de psicologia em relação a estresse e depressão, embora tenham encontrado níveis relativamente baixos de depressão entre os alunos, apontaram a correlação entre estresse e depressão para a amostra total. Mesmo sem estudar eventos estressores acadêmicos de forma específica, no estudo 
de Aguiar e cols. (2009), 75\% dos pesquisados apontou que as tarefas acadêmicas eram fonte de estresse.

Os estudos específicos sobre percepção de estressores e coping apontam uma grande variabilidade de eventos como desencadeadores de estresse entre alunos, e apontam que as fontes de estresse podem ser bastante particulares dependendo do curso ou área de estudos. Nesse sentido, há estudos pontuais sobre aspectos estressores do curso de enfermagem (Evangelista, Hortense, \& Souza, 2004) e medicina (Furtado e cols., 2003), por exemplo. De forma geral, aspectos como falta ou desorganização do tempo (Bondan \& Bardagi, 2008; Furtado e cols., 2003; Santos \& Alves Júnior, 2007) e a falta de motivação (Bondan \& Bardagi, 2008; Santos \& Alves Júnior, 2007) são citados como bastante estressantes. Outros aspectos que costumam ser percebidos como estressores são o excesso de provas e trabalhos, a existência de conflitos com colegas e/ou professores, o mau desempenho, problemas financeiros na aquisição de materiais, equipamentos ou outras necessidades, falta de ligação dos conteúdos com a prática, entre outros. Ao construir um instrumento para avaliação de estresse entre estudantes de enfermagem, Costa e Polak (2009) referem que as atividades geradoras de estresse costumam se agrupar em seis domínios: realização das atividades práticas; comunicação profissional; gerenciamento do tempo; ambiente; formação profissional e atividade teórica.

O estudo de Bondan e Bardagi (2008) comparou a quantidade de estressores percebidos (através de uma escala de estressores acadêmicos) entre alunos de cursos tecnológicos e regulares e também as diferenças de gênero. Não houve diferenças na quantidade de estressores percebidos entre homens e mulheres; já os alunos de cursos tecnológicos perceberam significativamente mais estressores do que os alunos de cursos regulares. Para os autores, essa diferença pode ser atribuída às diferenças de perfil entre os dois grupos (alunos dos cursos tecnológicos são mais velhos, a maioria com família própria, já inseridos no mercado de trabalho e provavelmente afastados dos estudos há mais tempo); dessa forma, a estrutura da universidade, as exigências acadêmicas e a rotina da graduação talvez tragam maiores dificuldades para estes alunos.

Quanto ao coping, Zonta, Robles e Grosseman (2006) avaliaram a qualidade de vida de estudantes de medicina e identificaram as principais estratégias de coping utilizadas por eles. Os resultados apontaram que entre as principais estão a valorização dos relacionamentos interpessoais, o equilíbrio entre estudo e lazer, a organização do tempo, os cuidados com a saúde, alimentação e o sono, a prática de atividade física, a religiosidade e, por último, a procura por assistência psicológica. Estas estratégias podem ser consideradas mais como focalizadas na emoção do que no problema. Os autores salientam a importância de serviços de apoio pedagógico e psicológico aos estudantes e treinamento de enfrentamento do estresse durante a graduação. Santos e Alves Junior (2007) avaliaram estudantes de pós-graduação da área de saúde e ao identificarem as estratégias de coping utilizadas, verificaram que o foco no problema foi a estratégia mais utilizada pelos participantes que não tiveram estresse, principalmente para os homens, enquanto que no grupo com estresse não houve o predomínio de uma determinada estratégia de enfrentamento.

\section{DISCUSSÃO}

Percebe-se, a partir desta revisão da literatura, que ainda são muito recentes e escassos os estudos sobre estresse e coping entre universitários no Brasil, e que a maioria foca um grupo específico de estudantes, os da área da saúde. Pode-se pensar que essa concentração se justifica como uma extensão dos estudos que apontam os profissionais da saúde como bastante vulneráveis ao estresse (Telles \& Pimenta, 2009), aumentando a preocupação com estes profissionais ainda na fase da formação. Ainda, de forma geral, esses estudos utilizaram amostras relativamente pequenas e, com poucas exceções, avaliações mais qualitativas dos eventos estressores. Dessa forma, os resultados obtidos são bastante pontuais e não pretendem ser generalizados para a totalidade dos alunos universitários brasileiros, que configuram um grupo bastante heterogêneo. Assim, percebe-se claramente a necessidade de ampliar o escopo das pesquisas para um número maior e mais diversificado de alunos, a fim de obter um levantamento extensivo dos eventos considerados geradores de estresse no ambiente universitário, especificamente aqueles eventos que sejam próprios da rotina e experiência acadêmica. Como apontam Aguiar e cols. (2009), a maioria dos alunos indica as tarefas acadêmicas como geradoras de estresse; no entanto, não se conhece exatamente a natureza destas tarefas e a intensidade do desconforto que elas provocam. Além disso, identificar também o perfil dos alu- 
nos mais afetados pelos eventos estressores também é importante, para a criação de estratégias preventivas mais efetivas.

A vulnerabilidade do aluno universitário ao estresse, indicada por estudos internacionais (Martínez \& Pinto, 2005; Martínez, Pinto, \& Silva, 2000; Misra \& Mckean, 2000; Ross e cols., 1999) foi confirmada nos resultados dos estudos encontrados nesta revisão, em que os índices de presença de estresse são iguais ou superiores a $40 \%$ das amostras pesquisadas. Ainda, a correlação positiva entre estresse e depressão (Baptista \& Campos, 2000) enfatiza o potencial prejuízo à saúde mental e ao aproveitamento da experiência acadêmica. Esses resultados parecem confirmar a complexidade e o desafio que as tarefas do ambiente universitário impõem aos alunos (Almeida \& Soares, 2003) e que, talvez, para muitos, possam ser vivenciadas como excessivamente ansiogênicas ou incompatíveis com os recursos pessoais percebidos, o que configuraria a avaliação do potencial estressor das mesmas (Lazarus \& Folkman, 1984).

A ampliação da avaliação sobre barreiras ou estressores percebidos configura-se como um instrumento interessante de investigação sobre as vivências acadêmicas, pois pode permitir a identificação de um contexto de maior ou menor fragilização da relação do aluno com o curso, as pessoas e a instituição. Como apontam os estudos internacionais, quanto maior a identificação do aluno com a instituição e a área de formação e melhores recursos de coping ele possuir, menor vai ser o impacto de eventuais problemas ao longo da trajetória (Eagan \& Walsh, 1995; Heppner e cols., 1995; Misra \& McKean, 2000; Robitschek \& Cook, 1999). Na perspectiva do desenvolvimento de carreira, o aluno que está satisfeito com a carreira e o curso que frequienta e consegue elaborar metas em longo prazo dentro desta carreira pode ser mais tolerante com eventos negativos que, para outro aluno pouco envolvido com o curso e a instituição, serão mais desgastantes e difíceis de superar. Gonçalves e Benevides-Pereira (2009) também indicam que o compromisso com a carreira pode ser um moderador do estresse, o que reforça a importância do componente vocacional neste contexto. Muitos dos problemas e dificuldades percebidos pelos estudantes podem ser reflexos de um desconforto em relação à própria escolha profissional. Essa diferenciação, entre estressores percebidos por alunos engajados e desengajados com o curso, ainda não foi objeto de estudo no contexto brasileiro e requer maiores investigações. Ainda, mais pesquisas avaliando as relações entre percepção de estressores, coping e personalidade podem auxiliar na identificação de quais alunos são mais vulneráveis às dificuldades acadêmicas.

Por outro lado, ao pensarmos pela ótica do curso, a avaliação e o feedback constante para a instituição sobre os problemas que afetam o aluno universitário podem ser de extrema utilidade para os membros das unidades de ensino repensarem suas estratégias de ação e proporem soluções focais para essas questões. Ao pensarmos que há problemas específicos que afetam alunos das diferentes áreas e níveis (Evangelista e cols., 2004; Santos \& Alves Junior, 2007; Zonta e cols., 2006), soluções também pontuais podem ser implementadas dentro das próprias unidades. A abordagem transacional do estresse de Lazarus e Folkman (1984) indica o aspecto contextual como central da avaliação e intervenção. Dessa forma, seria importante que as estratégias de intervenção investissem no desenvolvimento de recursos de enfrentamento nos alunos (recursos pessoais, maior autoconfiança, construção de redes de apoio, avaliação da dimensão e relevância dos problemas) (Heppner e cols., 1995; Robitschek \& Cook, 1999) a fim de que estas situações não atinjam proporções que diminuam o engajamento do aluno com o curso e a carreira, e que estas intervenções sejam criadas a partir de informações próprias do contexto de estudo e prática vivenciados pelos alunos. Nesse sentido, os estudos nacionais encontram-se na direção esperada, de identificar problemas focais, de áreas e grupos de alunos específicos, o que facilitaria a criação de intervenções justamente direcionadas a estes problemas. Investigações detalhadas sobre as particularidades dos diferentes grupos de estudantes universitários podem fornecer subsídios importantes para a criação de intervenções e serviços de apoio que venham realmente ao encontro das demandas do aluno, e não sejam apenas generalizações de intervenções criadas para outros públicos e settings adaptadas ao contexto universitário.

Os achados dos estudos nacionais revisados também confirmam a maior vulnerabilidade feminina ao estresse, citada na literatura internacional com universitários (Lent e cols., 2002; Misra \& McKean, 2000) e também na literatura nacional com profissionais já inseridos no mercado (Rosseti e cols., 2008). Ao avaliarem a quantidade de eventos estressores, Bondan e Bardagi (2008) não encontraram diferenças entre ho- 
mens e mulheres. No entanto, como a maioria dos estudos era com estudantes da área de saúde, em que predominam as mulheres, à exceção justamente do estudo de Bondan e Bardagi (2008), esses resultados devem ser vistos com cautela. Novos estudos com amostras maiores e mais diversificadas podem auxiliar na compreensão das diferenças de gênero na percepção e no enfrentamento dos eventos estressores.

Os poucos estudos que investigaram as estratégias de coping utilizadas pelos alunos vão ao encontro da literatura internacional, que costuma citar uma predominância de coping emocional/reativo e uma deficiência de coping reflexivo e busca de ajuda entre os estudantes (Jackson \& Finney, 2002; Lent e cols., 2002; Misra \& McKean, 2000; Robitschek \& Cook, 1999). Nas pesquisas descritas nesta revisão, também houve prevalência de coping focado na emoção. A dificuldade em enfrentar os problemas acadêmicos ou a demora em admiti-los já havia sido observada em estudos na área do desenvolvimento vocacional (Bardagi, 2007; Lehman, 2005, entre outros), indicando que o período da graduação merece atenção tanto dos profissionais e pesquisadores da área de carreira quanto de saúde mental em geral.

Espaços institucionais para a discussão e a solução dos problemas enfrentados pelos alunos são necessários para que a experiência acadêmica seja vivenciada não de forma isolada, mas como um processo que envolve os alunos e também os professores e a instituição como um todo. Serviços de apoio ao aluno podem criar intervenções favorecedoras do coping reflexivo, como sugerem Robitschek e Cook (1999), uma vez que este tipo de estratégia (focada na tentativa de resolução dos problemas percebidos) tem se mostrado associado ao maior ajustamento acadêmico (Crockett e cols., 2007). É importante que outros estudos sobre estresse e coping ampliem o escopo de informações sobre as relações entre a percepção de dificuldades, as estratégias de enfrentamento disponibilizadas pelos alunos e as conseqüências para o desenvolvimento pessoal e de carreira durante a graduação.

\section{REFERÊNCIAS}

Obs.: Referências precedidas de um asterisco indicam estudos incluídos na revisão.

*Aguiar, S. M., Vieira, A. P. G. F., Vieira, K. M. F., Aguiar, S. M., \& Nóbrega, J. O. (2009). Prevalência de sintomas de estresse nos estudantes de medicina. Jornal Brasileiro de Psiquiatria, 58, 34-38.
Almeida, L. S., \& Soares, A. P. (2003). Os estudantes universitários: Sucesso escolar e desenvolvimento psicossocial. In: E. Mercuri \& S. A. J. Polydoro (Orgs.), Estudante universitário: Características e experiências de formação (pp.15-40). Taubaté, SP: Cabral.

*Baptista, M. N., \& Campos, L. F. L. (2000). Avaliação longitudinal de sintomas de depressão e estresse em estudantes de psicologia. Boletim de Psicologia, 50, 37-58.

Bardagi, M. P. (2007). Evasão e comportamento vocacional de universitários: Estudos sobre o desenvolvimento de carreira na graduação. Tese de doutorado não-publicada, Universidade Federal do Rio Grande do Sul. Porto Alegre.

*Bondan, A. P., \& Bardagi, M. P. (2008). Comprometimento profissional e estressores percebidos por graduandos regulares e tecnológicos. Paidéia, 18, 581-590.

*Calais, S. L., Andrade, L. M. B., \& Lipp, M. E. N. (2003). Diferenças de sexo e escolaridade na manifestação de stress em adultos jovens. Psicologia: Reflexão e Crítica, 16, 257-263.

*Calais, S. L., Carrara, K., Brum, M. M., Batista, K., Yamada, J. K., \& Oliveira, J. R. S. (2007). Stress entre calouros e veteranos de jornalismo. Estudos de Psicologia (Campinas), 24, 6977.

Chaves, E. C., Cadê, N. V., Montovani, M. F., Leite, R. C. B., \& Spire, W. C. (2000). Coping: Significados, interferência no processo saúde-doença e relevância para a enfermagem. Revista da Escola de Enfermagem da USP, 34, 370-375.

*Costa, A. L. S., \& Polak, C. (2009). Construção e validação de instrumento para avaliação de estresse em estudantes de enfermagem (AEEE) [Número especial]. Revista da Escola de Enfermagem da USP, 43, 1017-1026.

Crockett, L. J., Iturbide, M. I., Torres-Stone, R. A., McGinley, M., Raffaelli, M., \& Carlo, G. (2007). Acculturative stress, social support, and coping: Relations to psychological adjustment among Mexican American college students. Cultural Diversity and Ethnic Minority Psychology, 13, 347-355.

Dela Coleta, A. S. M., \& Dela Coleta, M. F. (2008). Fatores de estresse ocupacional e coping entre policiais civis. PsicoUSF, $13,59-68$.

Dell'Aglio, D. D., \& Hutz, C. S. (2002). Estratégias de coping de crianças e adolescentes em eventos estressantes com pares e com adultos. Psicologia USP, 13, 203-225.

Eagan, A. E., \& Walsh, W. B. (1995). Person-environment congruence and coping strategies. Career Development Quarterly, 43, 246- 256.

*Evangelista, R. A., Hortense, P. F., \& Sousa. A. E. F. (2004). Estimação de magnitude do estresse pelos alunos de graduação, quanto ao cuidado de enfermagem. Revista Latino-Americana de Enfermagem, 12, 913-917.

Folkman, S., \& Lazarus, R. S. (1980). An analysis of coping in a middle-aged community sample. Journal of Health and Social Behavior, 21, 219-239.

Folkman, S., Lazarus, R., Gruen, R., \& De Longis, A. (1986). Appraisal, coping, health status and psychological symptoms. Journal of Personality and Social Psychology, 50, 571-579.

*Furtado, E. S., Falcone, E. M. O., \& Clark, C. (2003). Avaliação do estresse e das habilidades sociais na experiência acadêmica de estudantes de medicina de uma universidade do Rio de Janeiro. Interação em Psicologia, 7, p. 43-51. 
*Gonçalves, M. B., \& Benevides-Pereira, A. M. T. (2009). Considerações sobre o ensino médico no Brasil: Consequências afetivo-emocionais nos estudantes. Revista Brasileira de Educação Médica, 33, 493-504.

Hansen, D., \& Jarvis, P. (2000). Adolescent employment and psychosocial outcomes. Youth and Society, 31, 417-436.

Heppner, P. P., Cook, S. W., Wright, D. M., \& Johnson, W. C., Jr. (1995). Progress in resolving problems: A problem-focused style of coping. Journal of Counseling Psychology, 42, 279293.

Jackson, P. B., \& Finney, M. (2002). Negative life events and psychological distress among young adults. Social Psychology Quarterly, 65, 186-201.

Jo Lohman, B., \& Jarvis, P. (2000). Adolescent stressors, coping strategies, and psychological health studied in the family context. Journal of Youth and Adolescence, 29, 15-41.

*Katsurayama, M., Becker, M. A. D., Gomes, N. M., Santos, M. C., Makimoto, F. H., \& Santana, L. L. O. (2009). Fatores de risco e proteção em estudantes de medicina da Universidade Federal do Amazonas. Psicología para America Latina, 16. Retirado de http://www.psicolatina.org/16/fatores.html

Lazarus, R., \& Folkman, S. (1984). Stress, appraisal and coping. New York: Springer.

Lehman, Y. P. (2005). Estudo sobre evasão universitária: As mudanças de paradigmas na educação e suas conseqüências. Tese de livre docência não-publicada, Universidade de São Paulo.

Lent, R. W., Brown, S. D., Talleyrand, R., McPartland, E. B., Davis, T., Chopra, S. B., Suthakaran, V., \& Chai, C. (2002). Career choice barriers, supports, and coping strategies: College students' experiences. Journal of Vocational Behavior, 60, 6172 .

Lipp, M. E. N., \& Tanganelli, M. S. (2002). Stress e qualidade de vida em magistrados da justiça do trabalho: Diferenças entre homens e mulheres. Psicologia: Reflexão e Crítica, 15, 537548.

Martinez, I. M. M., \& Pinto, A. M. (2005). Burnout en estudiantes universitarios de España y Portugal y su relación con variables académicas. Aletheia, 21, 21-30.

Martinez, I. M. M., Pinto, A. M., \& Silva, A. L. (2000). Burnout em estudantes do ensino superior. Revista Portuguesa de Psicologia, 35, 151-167.

Misra, R., \& McKean, M. (2000). College students' academic stress and its relation to their anxiety, time management and leisure satisfaction. American Journal of Health Studies, 16, 41-51.

Noronha, A. P. P., \& Fernandes, D. C. (2008). Estresse laboral: Análise da produção científica brasileira na SciELO e BVSPsi. Fractal: Revista de Psicologia, 20, 491-501.
Olah, A. (1995). Coping strategies among adolescents: A crosscultural study. Journal of Adolescence, 18, 491-512.

Piko, B. (2001). Gender differences and similarities in adolescents' ways of coping. The Psychological Record, 51, 223-235.

Pinheiro, F. A., Tróccoli, B. T., \& Tamayo, M. R. (2003). Mensuração de coping no ambiente ocupacional. Psicologia: Teoria e Pesquisa, 19, 153-158.

Robitschek. C., \& Cook, S. (1999). The influence of personal growth initiative and coping styles on career exploration and vocational identity. Journal of Vocational Behavior, 54, 127141.

Rodrigues, A. B., \& Chaves, E. C. (2008). Stressing factors and coping strategies used by oncology nurses. Revista LatinoAmericana de Enfermagem, 16, 24-28.

Ross, S. E., Niebling, B. C., \& Heckert, T. M. (1999). Sources of stress among college students. College Student Journal, 33, 312-317.

Rossetti, M. O., Ehlers, D. M., Guntert, I. B., Leme, I. F. A. S., Rabelo, I. S., Tosi, S. M. V. D., Pacanaro, S. V., \& Barrionuevo, V. L. (2008). O inventário de sintomas de stress para adultos de Lipp (ISSL) em servidores da Polícia Federal de São Paulo. Revista Brasileira de Terapias Cognitivas, 4, 108-120.

*Santos, A. F., \& Alves Jr., A. (2007). Estresse e estratégias de enfrentamento em mestrandos de Ciências da Saúde. Psicologia: Reflexão e Crítica, 20, 104-113.

Seidl, E. M. F., Tróccoli, B. T., \& Zannon, C. M. L. C. (2001). Análise fatorial de uma medida de estratégias de enfrentamento. Psicologia: Teoria e Pesquisa, 17, 225-234.

Suehiro, A. C. B., Santos, A. A. A., Hatamoto, C. T., \& Cardoso, M. M. (2008). Vulnerabilidade ao estresse e satisfação no trabalho em profissionais do Programa de Saúde da Família. Boletim de Psicologia, 58, 205-218.

Telles, H., \& Pimenta, A. M. C. (2009). Síndrome de burnout em Agentes comunitários de saúde e estratégias de enfrentamento. Saude \& Sociedade, 18, 467-478.

*Zonta, R., Robles, A. C. C., \& Grosseman, S. (2006). Estratégias de enfrentamento do estresse desenvolvidas por estudantes de medicina da Universidade Federal de Santa Catarina. Revista Brasileira de Educação Médica, 30, 147-153.
Recebido: 23/03/2010

Última revisão: 15/01/2011

Aceite final: 28/02/2011 